Available online at GSC Online Press Directory

GSC Biological and Pharmaceutical Sciences

e-ISSN: 2581-3250, CODEN (USA): GBPSC2

Journal homepage: https://www.gsconlinepress.com/journals/gscbps

(RESEARCH ARTICLE)

\title{
In silico screening and molecular docking of bioactive agents towards human coronavirus receptor
}

\author{
Pratyush Kumar *, Asnani Alpana, Chaple Dinesh and Bais Abhinav \\ Priyadarshini J. L. College of Pharmacy, Electronic Building, Electronic Zone, MIDC, Hingna Road, Nagpur-440016, \\ Maharashtra, India.
}

Publication history: Received on 09 April 2020; revised on 13 April 2020; accepted on 15 April 2020

Article DOI: https://doi.org/10.30574/gscbps.2020.11.1.0099

\begin{abstract}
Coronavirus infection has turned into pandemic despite of efforts of efforts of countries like America, Italy, China, France etc. Currently India is also outraged by the virulent effect of coronavirus. Although World Health Organisation initially claimed to have all controls over the virus, till date infection has coasted several lives worldwide. Currently we do not have enough time for carrying out traditional approaches of drug discovery. Computer aided drug designing approaches are the best solution. The present study is completely dedicated to in silico approaches like virtual screening, molecular docking and molecular property calculation. The library of 15 bioactive molecules was built and virtual screening was carried towards the crystalline structure of human coronavirus (6nzk) which was downloaded from protein database. Pyrx virtual screening tool was used and results revealed that F14 showed best binding affinity. The best screened molecule was further allowed to dock with the target using Autodock vina software. The results of docking of F14 with target using autodock vina revealed the binding affinity of -10.6. The interaction study with discovery studio visualize revealed the molecular interaction with serine, histidine, asparagine, leucine, tyrosine, lysine, isoleucine, threonine. The molecular properties of F14 were also calculated. The study helped us understand that 3-amino-2-phenylquinazolin$4(3 \mathrm{H})$-one molecule can act as a potent parent structure for treating pandemic of coronavirus. This might be an essential tool for the medicinal chemist for designing novel molecule for treatment of coronavirus infection.
\end{abstract}

Keywords: Coronavirus; Virtual screening; Molecular docking; Autodock vina; Molinspiration

\section{Introduction}

Respiratory disorders are very common in country like India. The major reason for such disorders is pollution. The emissions from automobiles and several industries are degrading the quality of air. Currently the world is fighting against coronavirus disease [1].The origin of this virus is from china and has currently spread all over the world. COVID19 is an infectious agent responsible for the disease. The coronavirus has entered the periphery of Indian borders through several migrants. The contagious and infectious nature of the virus has lead to spreading among several individuals [2].

People infected with the COVID-19 virus experiences mild to moderate respiratory illness, older people, and those with underlying medical problems like cardiovascular disease, diabetes, chronic respiratory disease, and cancer are more likely to develop serious illness.

At this time, there are no specific vaccines or treatments for COVID-19. However, there are many ongoing clinical trials evaluating potential treatments. WHO will continue to provide updated information as soon as clinical findings become available [3].

\footnotetext{
* Corresponding author: Pratyush Kumar
} 
The exposure of corona virus has become very virulent in december 2019 and till date i.e. march 2020 and has claimed several lives worldwide. The number of infected persons isincreasing but still there are no vaccines or ideal medicines developed world wide for treating infection. Many countries have also lost control on this pandemic. The world health organization (WHO) has issued several guidelines which only includes details of prophylactic therapy. This prophylactic therapy includes sanitization as a key measure [4].

Computer aided drug designing is an innovative approach which utilizes in silico techniques to minimize the time and efforts in the pipeline of drug discovery. These techniques include study of the protein structure responsible for the disease or disorder, then identifying a ligand molecule that might have potent activities. Molecular docking techniques are study of interaction of the ligand with the protein molecule at active site. The active site is that three dimensional site of protein molecule which directly effects the activity of the protein. Although the activity can be either positive or negative but still it helps medicinal chemist to proceed on rational path [5-6].

The interaction ligands at receptor binding sites are measured as binding affinity. The less is binding affinity the more efficiently to the receptor at prescribed site. The charmm force fields are utilized for calculating these values. Although the values vary marginally differ from the system to system and software to software. This is due the difference in the genetic algorithm of the systems.

\section{Material and methods}

\subsection{Softwares and programs}

\subsubsection{Chemsketch}

Chemsketch a chemical molecule drawing tool was used to draw the ligand compound [7]. This tool enables us to draw chemical molecules and save them directly in several formats like .mol, .jpg, .png and many more formats. We can also generate the international union of pure and applied chemistry (IUPAC) of the chemical structures. This software also help us for generation of simplified molecular input line entry system (SMILES) of the desired chemical structure.

\subsubsection{Avogadro software}

Avogadro software was used to convert the .mol file to .pdb format [8]. This is again open source software that helps optimize the chemical structure. This also helps in minimizing the energy which is very important protocol for in silico studies. The software also allows generation of structure through SMILES or by drawing tool. These chemical structures were saved in the format of .pdb format which is required for docking purpose.

\subsubsection{Pyrx software}

Pyrx software was used for virtual screening of library of derivatives [9]. The pyrx software is a open softwarefor virtual screening. The approach includes blasting of several ligand molecules to a target and segregate the best fit molecules from the library. The tool includes a 4 step protocol and can screen a big library of molecules simultaneously on a defined site of target/receptor. The results can easily be exported in microsoft excel format as .csv file.

\subsubsection{Autodock Vina software}

Autodock vina software[10]was used to carry molecular docking and check the interactions. Autodock is a preliminary docking program and is used for the semi-flexible protein ligand docking studies. The software helps us to generate top 10 conformations of ligands which is arranged in decreasing order. The conformation at first position is said to be the best comformation with root mean square deviation (RMSD) value to be zero.

The crystalline structure of human coronavirus was downloaded from protein database and the pdb code was [PDB: $6 n z k][11-12]$. Discovery studio 3.5 visualizer (DS visualizer) was used for molecular interaction and visualization [13].

\subsubsection{Molinspiration online property calculator}

Molinspiration online property calculator [14] was used to study the chemical properties of the compound. The best molecule which was screened from the library by Pyrx was docked using autodock software. The properties of the best molecule were calculated using online server of molinspiration software. 


\subsection{Preparation of ligand}

Ligand structure was drawn using Chemsketch software and the structure was cleaned using the clean structure tool. The structure was saved in the working folder as .mol file in a working folder. The .mol file present in working folder was then accessed in Avogadro software and structure was optimized using optimization tool. The optimized structure was exported in the working folder as F1.pdb - F15.pdb file format.

\subsection{Preparation of receptor}

The crystal structure of human coronavirus was downloaded in .pdb format from the online database. The ligands which were previously attached at the active sites were removed using DS visualize. The treated target was then saved in the working folder as target.pdb.

\subsection{Virtual Screening through Pyrx}

Pyrx software was used to carry out the virtual screening of developed library of 15 molecules F1-F15. The grid had following location

\section{Center}

X: 205.65 ;Y: 216.18 ;Z: 192.63

Dimension(Angstrom) $\quad$ X: $57.41 \quad ; Y: 60.80 \quad ; Z: 67.01$

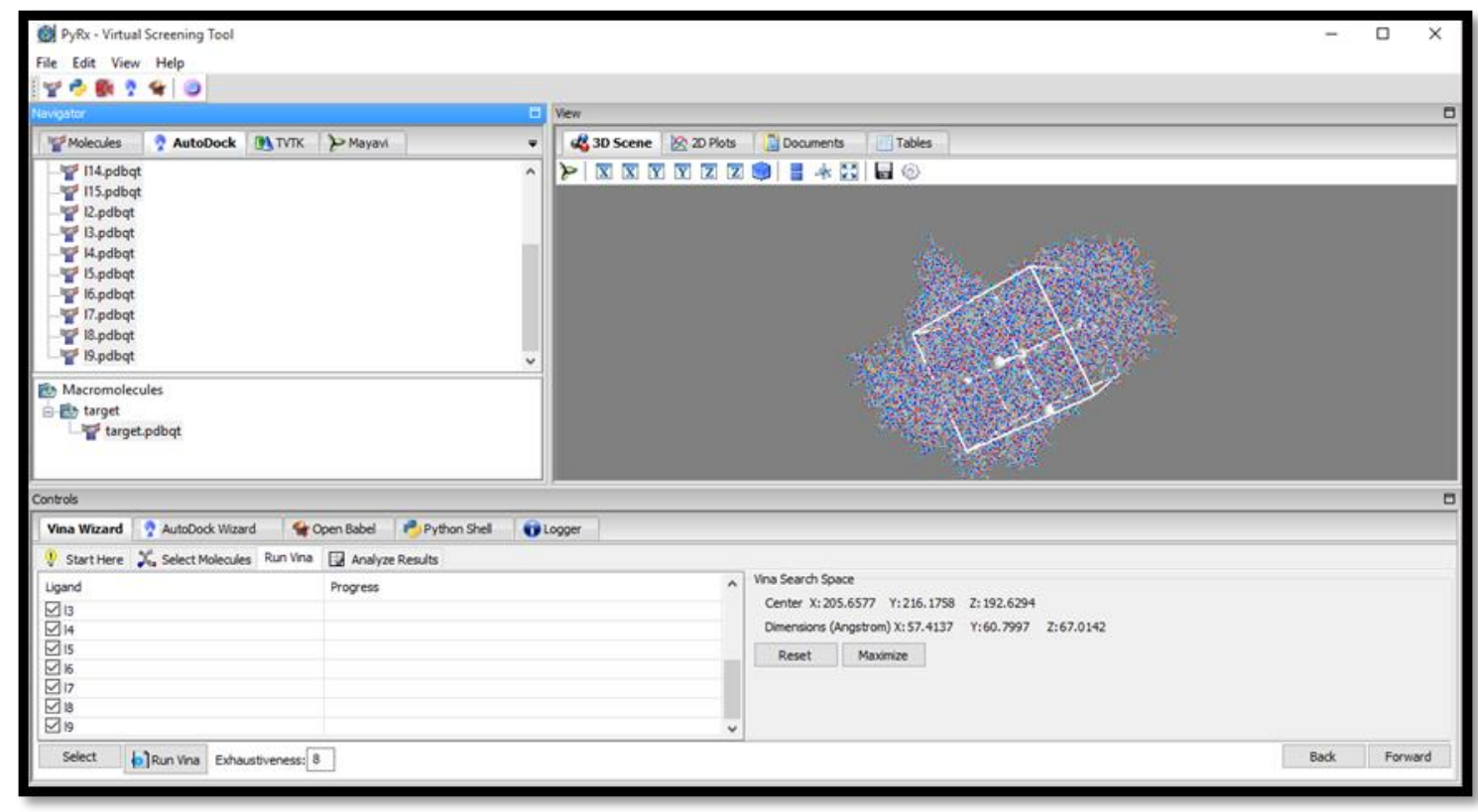

Figure 1 Window of Pyrx software with gridbox

The results are tabulated in table 1

\subsection{Receptor-Ligand Docking through Autodock vina}

Autodock vina was used to identify binding poses with associated binding energies. As per the inverse relation of energy and stability, the conformation with greater binding energy is less stable. The Default parameters of the software program have been applied similar to the protocol followed elsewhere. The ligand and target molecule were converted into the .pdbqt format. Briefly, Lamarckian Genetic Algorithm (LGA) with default atomic salvation parameters $126 \AA$ (x, $y$, and $z$ ) grid box in ratio of

Center

$$
\text { X: } 205.65 ; Y: 216.18 ; Z: 192.63
$$

Dimension(Angstroms) X: $57.41 \quad$;Y: $60.80 \quad$; Z: 67.01 was selected with 0.375 angstroms grid points spacing. 


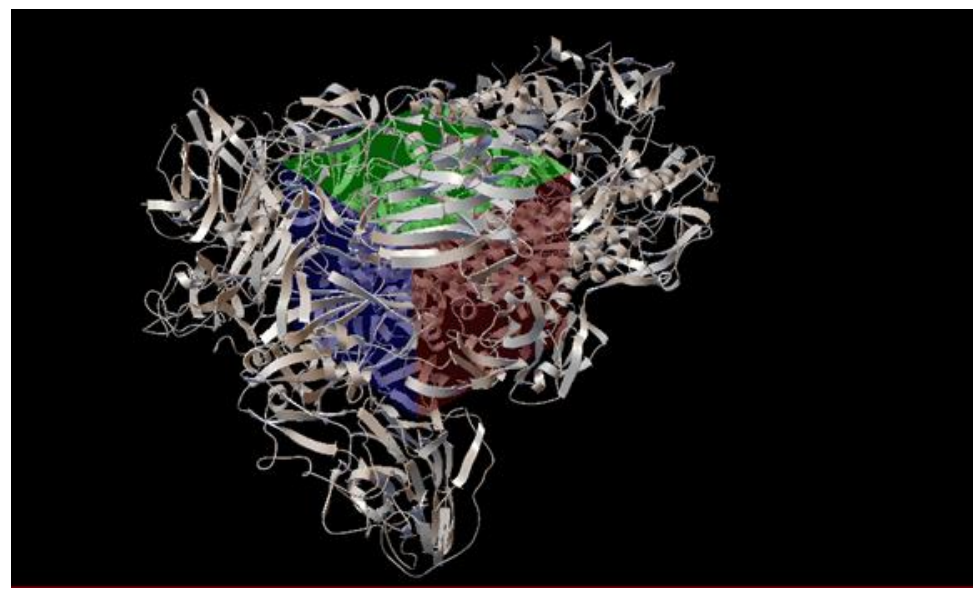

Figure 2Gridbox for docking using autodock

A conf.txt file was prepared with all essential command written and was saved in the working folder. Cygwin was used for running autodock vina and command [./vina.exe --config conf.txt.txt --log log.txt] was initiated for carrying out docking. The results of docking were saved in form of ligand_out.pdbqt format.The results of interactions were visualized using autodock software and DS visualizer.

\subsection{Online chemical property calculator}

Molinspiration online property calculator was used for calculating the properties of the ligand. The structure of the ligand was drawn using inbuilt tool and several properties were calculated. The properties were classified broadly into two types as structural property and bioactivity.

\section{Results and discussion}

\subsection{Virtual Screening}

The results of virtual screening are tabulated below. The interactions with RMSD zero was considered to be best and the binding affinities of F1-F15 molecules are represented in table 1.

Table 1 Results of Binding Affinity of Molecule

\begin{tabular}{lll}
\hline S.no. Name of the molecule & $\begin{array}{l}\text { Binding affinity } \\
\text { (Kcal/mol) }\end{array}$ \\
\hline F1 $\quad$ Benzene & Aniline
\end{tabular}




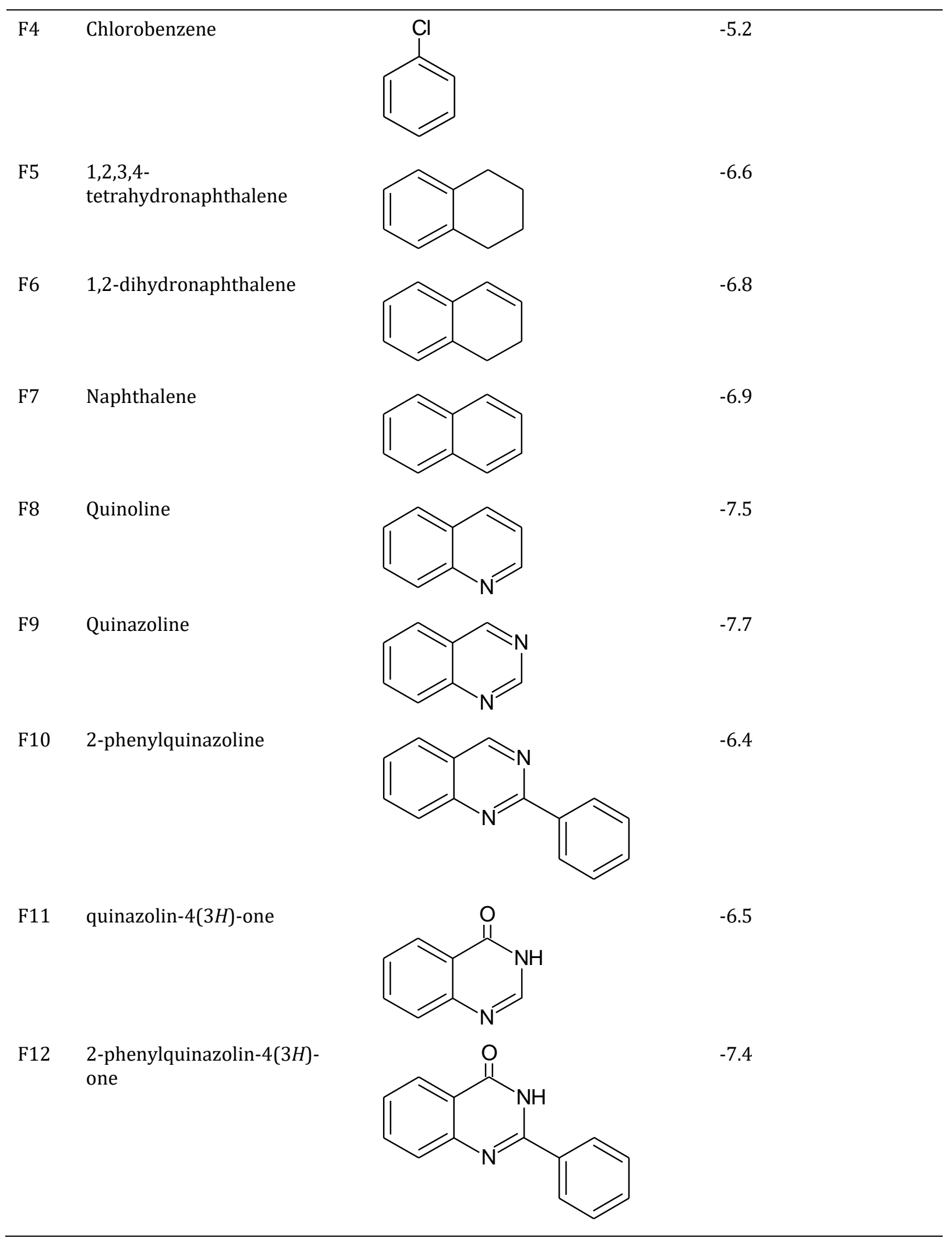


F13 3-hydroxy-2-

phenylquinazolin- $4(3 H)$-one<smiles>O=c1c2ccccc2nc(-c2ccccc2)n1O</smiles>

F14 3-amino-2-phenylquinazolin$4(3 H)$-one<smiles>Nn1c(-c2ccccc2)nc2ccccc2c1=O</smiles>

F15 2-phenyl-4H-3,1-benzoxazin4-one<smiles>O=c1oc(-c2ccccc2)nc2ccccc12</smiles>

$-7.2$

$-8.5$

\subsection{Molecular Docking}

The best molecule selected from the virtual screening was F14. The result of docking of F14 with the target molecule is shown in Figure 3.

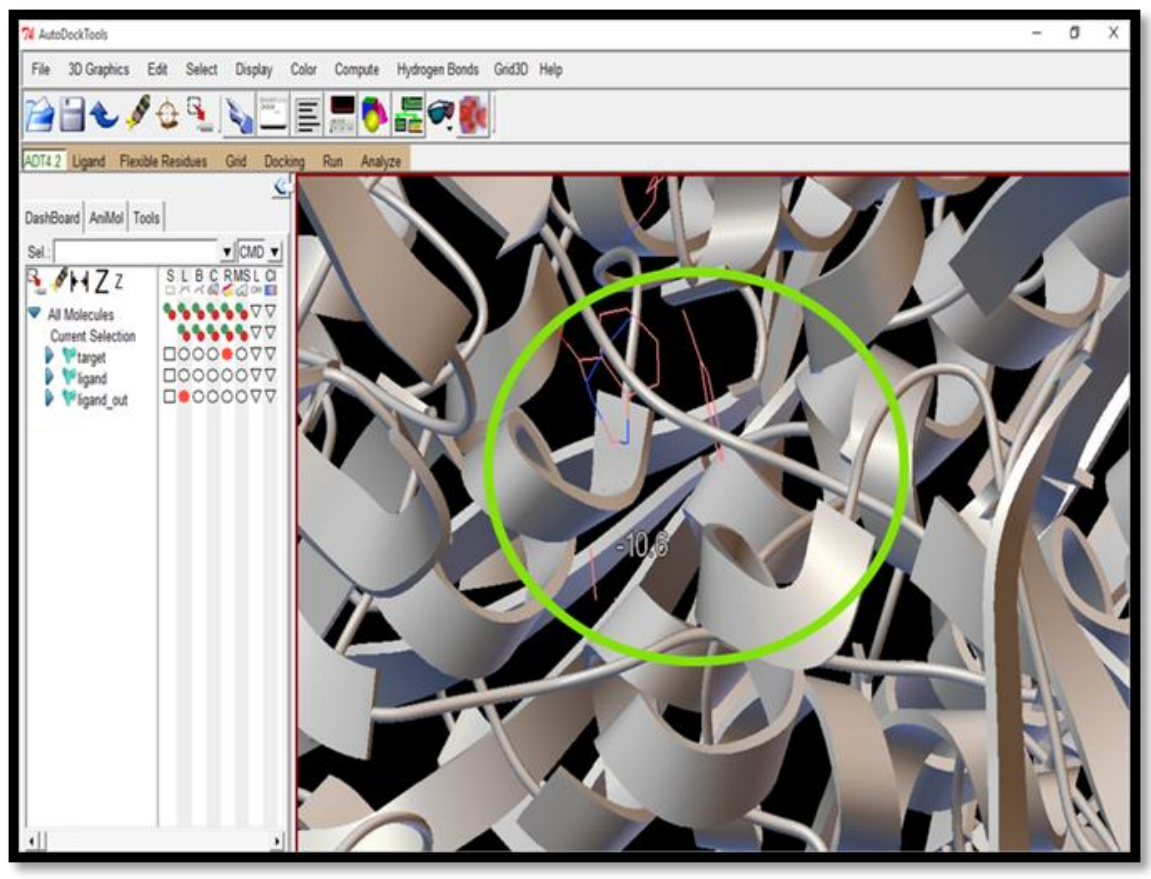

Figure 3 Docking of ligand with receptor showing binding affinity of -10.6 and having RMSD value of zero.

Further visualization of interactions of ligand molecule was carried out using DS visualizer and the result is shown in Figure 4. 


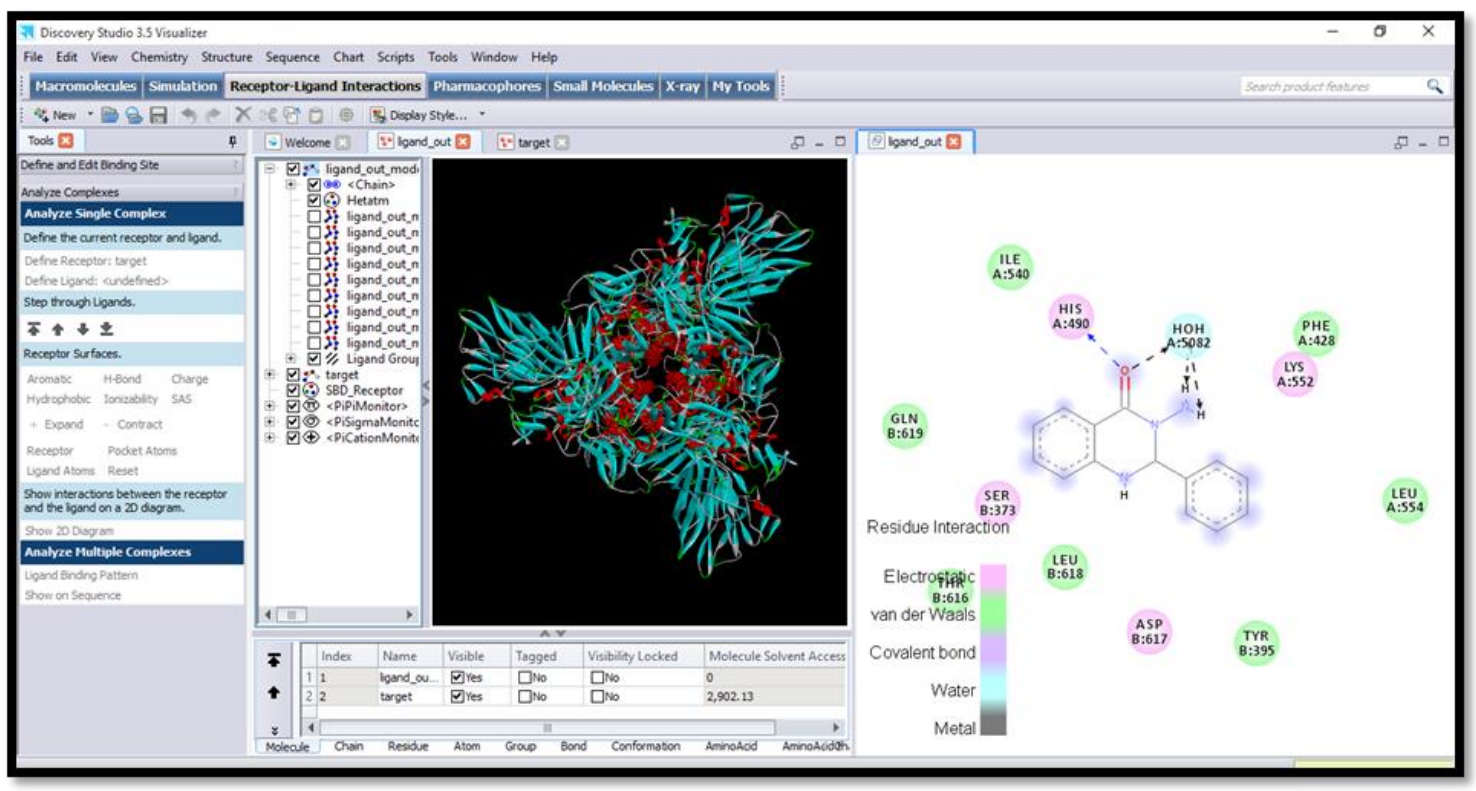

Figure 4 Interactions of ligand (F14) with target

The mean binding energy of the best conformation was found to be -10.6 and the root mean square deviation (RMSD)

value was found to be zero.

\subsubsection{Online chemical property calculator}

The results of molinspiration online property calculator were represented as structural property and predicted bioactivity in table 2 .

Table 2 Results of Molinspiration online property calculator for F14

\begin{tabular}{lllll}
\hline Sr. No & Structural Property & Predicated Bioactivity & \\
\hline & Property & Value & Site & Binding efficiency \\
\hline 1 & Milog P & 2.55 & GPCR Ligand & -0.47 \\
2 & TPSA & 60.92 & Ion channelmodulator & -0.47 \\
3 & Natoms & 18 & Kinase Inhibitor & -0.28 \\
4 & MW & 237.26 & Nuclear receptor ligand & -0.96 \\
5 & nON & 4 & Protease inhibitor & -0.85 \\
6 & nOHNH & 2 & Enzyme inhibitor & -0.22 \\
7 & Volume & 210.93 & & \\
\hline
\end{tabular}

\section{Conclusion}

In silico screening of biologically active ligands were carried out towards human coronavirus. The library of 15 molecules were prepared and results of virtual screening revealed that F14 i.e. 3-amino-2-phenylquinazolin-4(3H)-one showed best binding affinity of -8.5. The results of docking of F14 with target using autodock vina revealed the binding affinity of -10.6 which was good. The interaction study revealed the molecular interaction with serine, histidine, asparagine, leucine, tyrosine, lysine, isoleucine and threonine. The molecular properties of F14 were also calculated. The study helped us understand that 3-amino-2-phenylquinazolin-4(3H)-one molecule can act as a potent parent 
structure for treating pandemic of coronavirus. This might be an essential tool for the medicinal chemist [15] for designing novel molecule for treatment of coronavirus infection.

The lead molecule found to show best results can be further derivatized for development of potent bioactive agents. Invivo studies can also be carried out for understanding the actual nature of molecule.

\section{Compliance with ethical standards}

\section{Acknowledgments}

I acknowledge my mentor and guide Dr. (Mrs) Alpana Asnani for supporting and motivating me. I also acknowledge Dr. Dinesh Chaple sir, Principal of my institution for providing opportunities to learn and explore several aspects. I also thank management and other teaching faculties of institution alongwith students.

\section{Disclosure of conflict of interest}

The authors declare that there are no conflicts of interest.

\section{References}

[1] Drosten C, Chiu LL, Panning M, Leong HN, Preiser W, Tam JS, Gunther S,Kramme S, Emmerich P, Ng WL, Schmitz $\mathrm{H}$ and Koay ES. (2004). Evaluation of advanced reverse transcription-PCR assays and an alternative PCR target region for detection of severe acute respiratory syndrome-associated coronavirus. J. Clin. Microbiol, 42, 20432047.

[2] Drosten C, Gunther S, Preiser W, van der Werf S, Brodt HR, Becker S, RabenauH, Panning M, Kolesnikova L, Fouchier RA, Berger A, Burguiere AM, CinatlJ, Eickmann M, Escriou N, Grywna K, Kramme S,Manuguerra JC, Muller S, Rickerts V, Sturmer M, Vieth S, Klenk HD, Osterhaus AD, Schmitz H and Doerr HW. (2003). Identification of a novel coronavirus in patients with severeacute respiratory syndrome. N. Engl. J. Med. 348, 1967-1976.

[3] Lia van der Hoek, Krzysztof Pyrc, Maarten F Jebbink,Wilma Vermeulen-Oost, Ron J M Berkhout, Katja C Wolthers, Pauline M E Wertheim-van Dillen, Jos Kaandorp, Joke Spaargaren \& Ben Berkhout, (2004), Identification of a new human coronavirus. Nature Medicine. 10, 368-373.

[4] Du L, He Y, Zhou Y, Liu S, Zheng BJ and Jiang S. (2009). The spike protein of SARSCoV- a target for vaccine and therapeutic development. Nat. Rev. Microbiol, 7, 226-236.

[5] Sood N, Chaudhary S, Pardeshi T, Mujawar S, Deshmukh KB, Sheikh SA et al.(2015). In-silico study of small cell lung cancer based on protein structure and function: A new approach to mimic biological system, J Adv Pharm Technol Res, 6, 125-129.

[6] He, Y., Li, J., Jiang, S., (2006), A single amino acid substitution (R441A) in the receptor binding domain of SARS coronavirus spike protein disrupts the antigenic structure and binding activity. Biochem. Biophys. Res. Commun. 344, 106-113.

[7] Allen D. Hunter,(1997), ACD/ChemSketch 1.0 (freeware); ACD/ChemSketch 2.0 and its Tautomers, Dictionary, and 3D Plug-ins; ACD/HNMR 2.0; ACD/CNMR 2.0, J. Chem. Educ. 74(8), 905.

[8] Patrick Avery, Herbert Ludowieg, Jochen Autschbach, Eva Zurek, (2018). Extended Hückel Calculations on Solids Using the Avogadro Molecular Editor and Visualizer, J. Chem. Educ. 95(2), 331-337.

[9] Dallakyan S, Olson AJ., (2015). Small-molecule library screening by docking with PyRx, J. Chem. Educ. 1263, 243250.

[10] OTrott and A J Olson. (2010). AutoDock Vina: improving the speed and accuracy of docking with a new scoring function, efficient optimization and multithreading, Journal of Computational Chemistry, 31, 455-461.

[11] Hu H, Li L, Ka RY, Kou B, Wang Z, Zhang L, Zhang H, Hao Z, Tsui WH and Ni A. (2005). Screening and identification of linear B-cell epitopes and entry-blocking peptide of severe acute respiratory syndrome (SARS)-associated coronavirus using synthetic overlapping peptide library. J. Comb. Chem, 7, 648-656.

[12] Holmes KV.(2005). Structural biology adaptation of SARS coronavirus to humans. Science, 309, 1822-1823. 
[13] Qazi Mohd Sajid Jamal, Mohtashim Lohani, Mohd Haris Siddiqui, Mohd Haneef, Shailendra Kumar Gupta, and Gulshan Wadhwa, (2012). Molecular interaction analysis of cigarette smoke carcinogens NNK and NNAL with enzymes involved in DNA repair pathways: An in silico approach, J. Chem. Educ. 8(17), 795-800.

[14] Neeraj Kumar, Shashank Mishra, Chandrashekhar Sharma, Hamendra singh, (2016). Insilico ADME, Bioactivity and Toxicity Analysis of Some Selected Antimalarial Agents, IJAPBR, 2016; 1(5), 1-8.

[15] Bais A, Chaple D, Asnani A, Kumar P and Mohurle S. (2020). Green synthesis of pyranopyrazole using microwave assisted techniques. GSC Biological and Pharmaceutical Sciences, 10(2), 111-119.

\section{How to cite this article}

Pratyush K, Asnani A, Chaple D and Bais A. (2020). In silico screening and molecular docking of bioactive agents towards human coronavirus receptor. GSC Biological and Pharmaceutical Sciences, 11(1), 132-140. 